\title{
APPROXIMATION OF THE VIBRATION MODES OF A PLATE COUPLED WITH A FLUID BY LOW-ORDER ISOPARAMETRIC FINITE ELEMENTS*
}

\author{
ERWIN HERNÁNDEZ ${ }^{1}$
}

\begin{abstract}
We analyze an isoparametric finite element method to compute the vibration modes of a plate, modeled by Reissner-Mindlin equations, in contact with a compressible fluid, described in terms of displacement variables. To avoid locking in the plate, we consider a low-order method of the so called MITC (Mixed Interpolation of Tensorial Component) family on quadrilateral meshes. To avoid spurious modes in the fluid, we use a low-order hexahedral Raviart-Thomas elements and a non conforming coupling is used on the fluid-structure interface. Applying a general approximation theory for spectral problems, under mild assumptions, we obtain optimal order error estimates for the computed eigenfunctions, as well as a double order for the eigenvalues. These estimates are valid with constants independent of the plate thickness. Finally, we report several numerical experiments showing the behavior of the methods.
\end{abstract}

Mathematics Subject Classification. 65N15, 65N30, 74F10, 74H25.

Received: July 24, 2004. Revised: September 22, 2004.

\section{INTRODUCTION}

This paper deals with the numerical computation of the vibration modes of a fluid-structure interaction problem in a 3D-domain. This is a very important engineering problem (e.g. for treatment of noise in cars or planes). It is well known that a large amount of work has been devoted to this subject (see for example [17]).

We are interested in one of problems of this kind: to compute elastoacoustic vibrations when the structure is an elastic plate and the fluid is ideal and compressible, both with small displacements.

In the framework of plate theory, we consider the most commonly used model for describe small as well as moderately thin plates: Reissner-Mindlin equations. It is well known that standard finite element methods produce unsatisfactory result when applied to this model, even for the plate alone; this phenomenon is due to numerical locking. To avoid this drawback, some special method based on reduced integration or mixed interpolation has to be used. One of the most used methods of this type is the MITC (Mixed Interpolation of Tensorial Component) methods introduced by Bathe and Dvorkin in [4]. A great number of paper dealing with the mathematical analysis of this method have been published in the context of load problem (see for example $[2,3,9,10,20])$ and for the plate vibration spectral problem (see $[11,13])$.

Keywords and phrases. Reissner-Mindlin, MITC methods, fluid-structure interaction.

* Supported by USM 12.04.01 and FONDECYT (Chile) through grant No.1040341.

${ }^{1}$ Departamento de Matemática, Universidad Técnica Federico Santa María, Casilla 110-V, Valparaiso, Chile.

e-mail: erwin.hernandez@usm.cl

(c) EDP Sciences, SMAI 2004 
To determining the vibration modes of the fluid, usually the pressure is chosen as primary variable; however, for coupled systems, the use of displacement vector fields present some advantageous properties like, for example, that compatibility and equilibrium through the fluid-structure interface satisfy automatically. Though, it is well known that the displacement formulation suffers from the presence of zero-frequency spurious modes with no physical meaning. An alternative approach has been introduced and analyzed in [7] to avoid the spurious modes; it consists in the use of lowest-order Raviart-Thomas element. Non-existence of spurious modes and optimal error estimates for two dimensional fluid-structure problem have been proved in [7] and [18].

The problem of a plate coupled with a fluid have been mathematically analyzed in [12], by using DL3 triangular finite element for the plate and tetrahedral Raviart-Thomas element for the fluid. Optimal order error estimates for the eigenvalues and eigenvectors valid uniformly on the thickness parameter have been obtained in that reference and no spurious modes are present with this discretization.

In the case of plate alone, recently, in [13] has been proved optimal error estimates in order and regularity for lowest-order quadrilateral MITC4 finite element under some technical assumption. These result have been proved for triangular element in [11], however those proofs can not be extended straightforwardly, even for the case of rectangular meshes.

In this paper we have extended the results in [12] by considering a discretization of the coupled problem involving the quadrilateral MITC4 finite element for the bending of the plate (the original Bathe and Dvorkin's paper deals with this element) and lowest-order hexahedral Raviart-Thomas element (see [21]) for the fluid. On the fluid-plate interface a non-conforming coupling is used; i.e., equal normal displacement for the fluid and plate is imposed in a weak sense. Moreover, in order to get error estimates optimal in order and regularity we assume the hypothesis of convexity of the domain. Let us remark that although the isoparametrical finite element are the most used element in engineering applications (quadrilateral in 2D and hexahedral in 3D), no available result seems to exist for this case.

The rest of the paper is organized as follow. In Section 2 we introduce the spectral problem to describe the free vibration modes for the coupled system. In Section 3, we describe the finite element method to solve the problem. We prove optimal order error estimates for the approximation. In Section 5 we prove error estimates for the spectral plate-fluid vibration problem. Finally, in Section 6, we report some numerical experiments.

Throughout the paper we denote by $C$ a positive constant not necessarily the same at each occurrence, but always independent of the mesh-size and the plate thickness.

\section{Statement of the problem}

We consider the problem of determining the free vibration modes of a three-dimensional cavity enclosing and ideal inviscid barotropic fluid. The walls of this cavity are considered to be all rigid, except for one of them which is an elastic plate. Let $\Omega$ be the domain occupied by the fluid and $\Gamma \times\left(-\frac{t}{2}, \frac{t}{2}\right)$, that of the plate, where $\Gamma$ is its middle surface of the plate of constant thickness $t>0$.

We consider that $\Omega$ is a polyhedral convex three-dimensional domain. Its boundary $\partial \Omega$ is the union of the convex surfaces $\Gamma_{0}, \Gamma_{1}, \ldots, \Gamma_{J}$. We assume that $\Gamma_{0}$ is in contact with the plate, whereas the remaining surfaces are assumed to be perfectly rigid walls. We denote by $n$ the unit outward normal vector to $\partial \Omega$.

Throughout this paper we make use of the standard notation for Sobolev spaces $H^{k}(\Omega), H_{0}^{1}(\Gamma), H(\operatorname{div}, \Omega)$, $H_{0}($ rot, $\Gamma)$, etc. and their respective norms (see for instance [15]). We also denote $\mathcal{H}:=L^{2}(\Gamma) \times L^{2}(\Gamma)^{2} \times L^{2}(\Omega)^{3}$, $\mathcal{X}:=H_{0}^{1}(\Gamma) \times H_{0}^{1}(\Gamma)^{2} \times H(\operatorname{div}, \Omega)$ and $\|\cdot\|$ the product norm of the latter.

In that follows, we introduce the coupled problem. For more details see [12].

In order to describe the deformation of the plate, we consider the Reissner-Mindlin model, which is written in terms of the rotations $\beta=\left(\beta^{1}, \beta^{2}\right)$ of the fibers initially normal to the plate midsurface and the transverse displacement $w$ (see $[8,10])$. The following equations describe the dynamic response of the plate to a pressure load $q$ exerted on one of its faces with $(w, \beta) \in H_{0}^{1}(\Gamma) \times H_{0}^{1}(\Gamma)^{2}$ being such that

$$
t^{3} a(\beta, \eta)+\kappa t \int_{\Gamma}(\nabla w-\beta) \cdot(\nabla v-\eta)+t \int_{\Gamma} \rho_{\mathrm{P}} \ddot{w} v+\frac{t^{3}}{12} \int_{\Gamma} \rho_{\mathrm{P}} \ddot{\beta} \cdot \eta=\int_{\Gamma} q v \quad \forall(v, \eta) \in H_{0}^{1}(\Gamma) \times H_{0}^{1}(\Gamma)^{2}
$$


(see for instance [16]). In the previous equation, the double dot means second derivatives with respect to time, $\rho_{\mathrm{P}}$ is the density of the plate, $\kappa:=\frac{E k}{2(1+\nu)}$, where $E$ is the Young modulus, $\nu$ the Poisson ratio of the plate and $k$ a correction factor which is usually taken as $5 / 6$ (see [1] for a justification of the use of this coefficient); finally, $a$ is the bilinear form $H_{0}^{1}(\Gamma)^{2}$-elliptic defined by

$$
a(\beta, \eta):=\frac{E}{12\left(1-\nu^{2}\right)} \int_{\Gamma}\left[\sum_{i, j=1}^{2}(1-\nu) \varepsilon_{i j}(\beta) \varepsilon_{i j}(\eta)+\nu \operatorname{div} \beta \operatorname{div} \eta\right] .
$$

On the other hand, to describe the governing equations for the free small amplitude motions of an inviscid compressible fluid contained in $\Omega$, we consider the displacement formulation

$$
\int_{\Omega} \rho_{\mathrm{F}} \ddot{u} \cdot \phi+\int_{\Omega} \rho_{\mathrm{F}} c^{2} \operatorname{div} u \operatorname{div} \phi=-\int_{\Gamma} p \phi \cdot n
$$

where $p$ is the pressure, $u$ the displacement field, $\rho_{\mathrm{F}}$ the density and $c$ the acoustic speed of the fluid.

Since the fluid is considered inviscid, only the normal component of the displacement vanishes on the rigid part of the cavity boundary $\Gamma_{\mathrm{R}}:=\Gamma_{1} \cup \cdots \cup \Gamma_{J}$ :

$$
u \cdot n=0 \quad \text { on } \Gamma_{\mathrm{R}}
$$

Since the transverse displacement of the plate do not depend on the $z$-coordinate, it can be considered that $\Gamma$ (instead of $\Gamma_{0}$ ) is one of the components of $\partial \Omega$. Then, the interface condition reads

$$
u \cdot n=w \quad \text { on } \Gamma \text {. }
$$

Finally, in our coupled problem, the unique load $q$ exerted on the plate is the pressure $p$ of the fluid.

Then, the space of kinematically admissible displacements of the coupled system is

$$
\mathcal{V}:=\left\{(v, \eta, \phi) \in \mathcal{X}: \phi \cdot n=0 \text { on } \Gamma_{\mathrm{R}} \text { and } \phi \cdot n=v \text { on } \Gamma\right\}
$$

and, by adding (1) to $(2)$, for all $(v, \eta, \phi) \in \mathcal{V}$ we have

$$
t^{3} a(\beta, \eta)+\kappa t \int_{\Gamma}(\nabla w-\beta) \cdot(\nabla v-\eta)+\int_{\Omega} \rho_{\mathrm{F}} c^{2} \operatorname{div} u \operatorname{div} \phi=-t \int_{\Gamma} \rho_{\mathrm{P}} \ddot{w} v-\frac{t^{3}}{12} \int_{\Gamma} \rho_{\mathrm{P}} \ddot{\beta} \cdot \eta-\int_{\Omega} \rho_{\mathrm{F}} \ddot{u} \cdot \phi .
$$

The free vibration modes of this coupled problem are obtained by seeking harmonic in time solutions of (5). By so doing we obtain the following spectral problem (see for instance [17]):

Find $\lambda \in \mathbb{R}$ and $0 \neq(w, \beta, u) \in \mathcal{V}$ such that

$$
\begin{aligned}
t^{3} a(\beta, \eta)+\kappa t \int_{\Gamma}(\nabla w-\beta) \cdot(\nabla v-\eta)+\int_{\Omega} \rho_{\mathrm{F}} c^{2} \operatorname{div} u \operatorname{div} \phi & \\
& =\lambda\left(t \int_{\Gamma} \rho_{\mathrm{P}} w v+\frac{t^{3}}{12} \int_{\Gamma} \rho_{\mathrm{P}} \beta \cdot \eta+\int_{\Omega} \rho_{\mathrm{F}} u \cdot \phi\right) \forall(v, \eta, \phi) \in \mathcal{V},
\end{aligned}
$$

where $\lambda$ is the square of the angular vibration frequency.

As usual, when a displacement formulation is used for the fluid, $\lambda=0$ is a solution of this problem with eigenspace given by

$$
\mathcal{K}:=\{(0,0, \phi) \in \mathcal{V}: \operatorname{div} \phi=0 \text { in } \Omega \text { and } \phi \cdot n=0 \text { on } \partial \Omega\} .
$$


The eigenfunctions corresponding to non-zero eigenvalues belong to the orthogonal complement of $\mathcal{K}$ in $\mathcal{V}$ with respect to the symmetric bilinear form in the right hand side of (6). This orthogonal complement consist of the conservative displacement fields in the fluid, namely

$$
\mathcal{G}:=\left\{(v, \eta, \phi) \in \mathcal{V}: \phi=\nabla q \text { for some } q \in H^{1}(\Omega)\right\}
$$

with

$$
\|\phi\|_{1, \Omega} \leq C\left(\|v\|_{1 / 2, \Gamma}+\|\operatorname{div} \phi\|_{0, \Omega}\right) .
$$

We observe that $\mathcal{K}$ and $\mathcal{G}$ are also orthogonal with respect to the bilinear form in the left hand side of (6). Hence, to obtain the eigenpairs corresponding to non-zero eigenvalues we can seek the solution of problem (6) restricted to $\mathcal{G}$ (i.e., with $\mathcal{V}$ substituted by $\mathcal{G}$ ).

For the theorical analysis we consider

$$
\|(v, \eta, \phi)\|_{\bullet}:=\left(\|v\|_{1, \Gamma}^{2}+\|\eta\|_{1, \Gamma}^{2}+\|\operatorname{div} \phi\|_{0, \Omega}^{2}\right)^{1 / 2}
$$

which is a norm on $\mathcal{G}$ equivalent to $\|\cdot\|$ (see [12]).

On the other hand, in static problems, the loads are typically assumed to depend adequately on the thickness in order to obtain a family of problems with uniformly bounded solutions: volumetric forces are supposed to be proportional to $t^{3}$ and surface loads to $t^{2}$ (see for instance [8]). For this reason, we assume that the densities for both, fluid and solid, are related with the thickness of the plate as follows:

$$
\rho_{\mathrm{F}}=\hat{\rho}_{\mathrm{F}} t^{3}, \quad \rho_{\mathrm{P}}=\hat{\rho}_{\mathrm{P}} t^{2} .
$$

Hence, we consider the following rescaled problem, for the non-zero eigenvalues of (6):

Find $\lambda \in \mathbb{R}$ and $0 \neq(w, \beta, u) \in \mathcal{G}$ such that

$$
s_{t}((w, \beta, u),(v, \eta, \phi))=\lambda r_{t}((w, \beta, u),(v, \eta, \phi)) \quad \forall(v, \eta, \phi) \in \mathcal{G}
$$

with

and

$$
s_{t}((w, \beta, u),(v, \eta, \phi)):=a(\beta, \eta)+\frac{\kappa}{t^{2}} \int_{\Gamma}(\nabla w-\beta) \cdot(\nabla v-\eta)+\int_{\Omega} \hat{\rho}_{\mathrm{F}} c^{2} \operatorname{div} u \operatorname{div} \phi
$$

$$
r_{t}((w, \beta, u),(v, \eta, \phi)):=\int_{\Gamma} \hat{\rho}_{\mathrm{P}} w v+\frac{t^{2}}{12} \int_{\Gamma} \hat{\rho}_{\mathrm{P}} \beta \cdot \eta+\int_{\Omega} \hat{\rho}_{\mathrm{F}} u \cdot \phi
$$

We consider the operator $T_{t}$ defined by

$$
\begin{aligned}
T_{t}: \underset{\mathcal{H}}{\mathcal{H}} & \longrightarrow \mathcal{G} \\
(f, \theta, g) & \longmapsto(w, \beta, u)
\end{aligned}
$$

with $(w, \beta, u) \in \mathcal{G}$ being the solution of

$$
s_{t}((w, \beta, u),(v, \eta, \phi))=r_{t}((f, \theta, g),(v, \eta, \phi)) \quad \forall(v, \eta, \phi) \in \mathcal{G} .
$$

This problem is well posed and the operator turns out to be uniformly bounded on $t$ (see [12]). Furthermore, as a consequence of $(9), \mathcal{G}$ is compactly included in $\mathcal{H}$ and, therefore, $T_{t}: \mathcal{H} \longrightarrow \mathcal{H}$ is a compact operator. Moreover, since $s_{t}$ and $r_{t}$ are symmetric and semipositive definite, the spectrum of $T_{t}$, apart from $\mu=0$, consists of a sequence of positive finite multiplicity eigenvalues converging to zero. Note that $\lambda$ is an eigenvalue of (11) if and only if $\mu=\frac{1}{\lambda}$ is an eigenvalue of $T_{t}$ with the same multiplicity and corresponding eigenfunctions. 


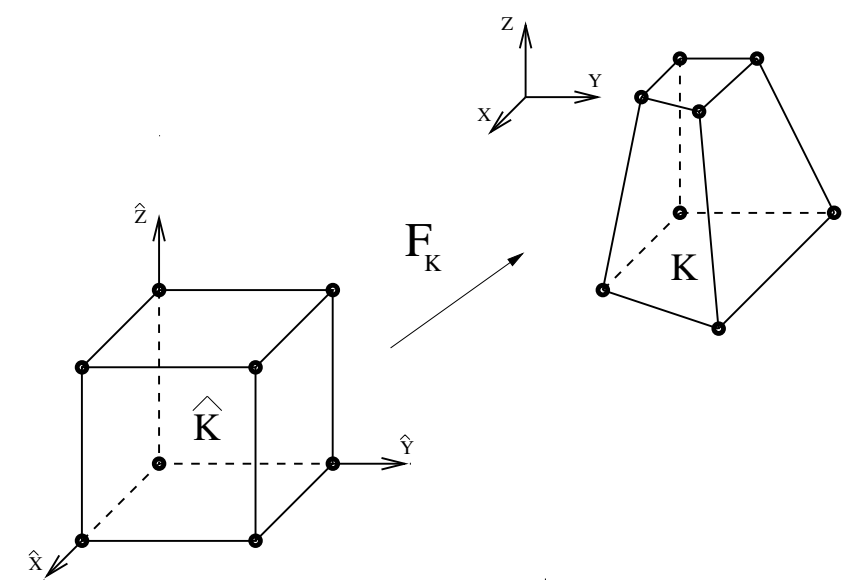

FiguRE 1. Trilinear mapping onto an element $\mathbf{K} \in \mathcal{T}_{h}$.

We will use the following regularity result for the solution of (12) (see [12]):

Theorem 2.1. Let $(f, \theta, g) \in \mathcal{H},(w, \beta, u)=T_{t}(f, \theta, g)$ and $\gamma=\frac{\kappa}{t^{2}}(\nabla w-\beta)$. Then, $(w, \beta, u) \in H^{2}(\Gamma) \times$ $H^{2}(\Gamma)^{2} \times H^{1}(\operatorname{div}, \Omega), \gamma \in L^{2}(\Gamma)$ and the following estimate holds

$$
\|w\|_{2, \Gamma}+\|\beta\|_{2, \Gamma}+\|u\|_{H^{1}(\operatorname{div}, \Omega)}+\|\gamma\|_{0, \Gamma} \leq C|(f, \theta, g)|_{t}
$$

with $C>0$ independent of $t$.

\section{Discretization}

Let $\left\{\mathcal{T}_{h}\right\}$ be a family of partitions in hexahedra of $\Omega$ and $\left\{\mathcal{T}_{h}^{\Gamma}\right\}$ be a family of decomposition of $\Gamma$ into convex quadrilaterals. Note that, although each $\mathcal{T}_{h}$ induces a decomposition on $\Gamma$, we do not assume that $\mathcal{T}_{h}^{\Gamma}$ is this induces meshes. That is each pair of meshes $\mathcal{T}_{h}$ and $\mathcal{T}_{h}^{\Gamma}$ do not need to be compatible. Here $h$ stands for the maximum diameter of the elements in $K \in \mathcal{T}_{h}^{\Gamma}$ or $\mathbf{K} \in \mathcal{T}_{h}$, respectively.

Let $\widehat{\mathbf{K}}:=[0,1]^{3}$ be the reference element for the partition $\mathcal{T}_{h}$. We denote by $Q_{i, j, k}(\widehat{\mathbf{K}})$ the space of polynomials of degree less than or equal to $i$ in the first variable, to $j$ in the second one, and to $k$ in the third one. Also, we set $Q_{k}(\widehat{\mathbf{K}})=Q_{k, k, k}(\widehat{\mathbf{K}})$. Similarly, for $\widehat{K}:=[0,1]^{2}$ the reference element for the partition $\mathcal{T}_{h}^{\Gamma}$, we define $Q_{i, j}(\widehat{K})$ and $Q_{k}(\widehat{K})$.

Let $\mathbf{K} \in \mathcal{T}_{h}$. We denote by $\mathbf{F}_{K}$ a trilinear mapping of $\widehat{\mathbf{K}}$ onto $\mathbf{K}$, with Jacobian matrix and determinant denoted by $D \mathbf{F}_{K}$ and $J_{\mathbf{F}_{K}}$ respectively (see Fig. 1). Analogously, let $K \in \mathcal{T}_{h}^{\Gamma}$, we denote by $F_{K}$ the bilinear mapping of $\widehat{K}$ onto $K$, with Jacobian $D F_{K}$ and determinant of the Jacobian $J_{F_{K}}$ (see Fig. 2).

We consider regular meshes in the sense that there exist constants $c$ and $C$ independent of the elements $K$ or $\mathbf{K}$ such that

$$
c h_{K}^{2} \leq J_{F_{K}} \leq C h_{K}^{2} \quad \forall K \in \mathcal{T}_{h}^{\Gamma}
$$

and

$$
c h_{\mathbf{K}}^{3} \leq J_{\mathbf{F}_{K}} \leq C h_{\mathbf{K}}^{3} \quad \forall \mathbf{K} \in \mathcal{T}_{h},
$$

respectively. 


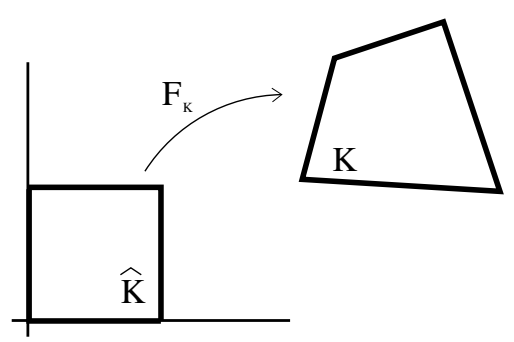

FIGURE 2. Bilinear mapping onto an element $K \in \mathcal{T}_{h}^{\Gamma}$.

Furthermore, according to $[13,14]$, we assume that the meshes are asymptotically paralelogramic, i.e. the above mapping verify

$$
\begin{gathered}
\frac{\left|\widehat{\nabla} J_{\mathbf{F}_{K}}\right|}{J_{\mathbf{F}_{K}}} \leq C h_{\mathbf{K}}, \\
\left|\widehat{\nabla}\left(J_{\mathbf{F}_{K}} D \mathbf{F}_{K}{ }^{-1}\right)\right| \leq C h_{\mathbf{K}}^{3},
\end{gathered}
$$

and

$$
\left|\widehat{\nabla}\left(D F_{K}^{-1}\right)\right| \leq C h_{K}^{2}
$$

for all $\mathbf{K} \in \mathcal{T}_{h}$ and $K \in \mathcal{T}_{h}^{\Gamma}$, respectively. Here, $|\cdot|$ denote the standard Euclidean norm and the corresponding matrix norm. Moreover, in the plate we assume that the mesh $\mathcal{T}_{h}^{\Gamma}$ is a refinement of a coarser partition $\mathcal{T}_{2 h}^{\Gamma}$, obtained by joining the midpoints of each opposite side in each $M \in \mathcal{T}_{2 h}^{\Gamma}$ (called macro-element). In addition, $\mathcal{T}_{2 h}^{\Gamma}$ is a similar refinement of a still coarser regular partition $\mathcal{T}_{4 h}^{\Gamma}$ (see [8]).

To approximate the fluid displacements, we use lowest order Raviart-Thomas elements (see [21]). Let

$$
\mathcal{R} T(\widehat{\mathbf{K}}):=\left\{\widehat{q}: \widehat{q} \in Q_{1,0,0}(\widehat{\mathbf{K}}) \times Q_{0,1,0}(\widehat{\mathbf{K}}) \times Q_{0,0,1}(\widehat{\mathbf{K}})\right\}
$$

and, from this space, we define through the contravariant transformation known as the Piola's transformation,

$$
\mathcal{R} T(\mathbf{K}):=\left\{q: q \circ \mathbf{F}_{K}=J_{\mathbf{F}_{K}}^{-1} D \mathbf{F}_{K} \widehat{q}, \quad \widehat{q} \in \mathcal{R} T(\widehat{\mathbf{K}})\right\} .
$$

Note that the Piola transform associated with $\mathbf{F}_{K}$ allows to transforming vector fields from the current element to the reference one (see the definition of the space $\mathcal{R} T(\widehat{K})$ ), leaving invariant its flux through any surface; i.e., for each face $S$ of $\mathbf{K}=\mathbf{F}_{K}(\widehat{\mathbf{K}})$ we have

$$
\int_{S} q \cdot n=\int_{\widehat{S}} \widehat{q} \cdot \widehat{n}
$$

where $n$ is the normal unit vector to $S$.

Then, we define the lowest-order Raviart-Thomas space (see [19,21])

$$
R_{h}:=\left\{\phi_{h} \in H(\operatorname{div}, \Omega):\left.\quad \phi_{h}\right|_{K} \in \mathcal{R} T(\mathbf{K}) \quad \forall \mathbf{K} \in \mathcal{T}_{h}\right\} .
$$

We remark that, for $R_{h} \subset H(\operatorname{div}, \Omega)$, the normal component of a function in $R_{h}$ must be continuous along interelement boundaries and vanish on $\partial \Omega$. In fact, the integrals (13) of these normal components are the degrees of freedom defining an element of $R_{h}$.

For the plate we consider a method MITC4, introduced by Bathe and Dvorkin (see [4]) and analyzed in $[3,10,13]$. It is based on different finite element spaces for the rotations, the transverse displacement and the shear strain. 
Let

$$
\mathcal{N}(\widehat{K}):=\left\{\widehat{p}: \widehat{p} \in Q_{0,1}(\widehat{K}) \times Q_{1,0}(\widehat{K})\right\}
$$

and, from this space, we define through covariant transformation:

$$
\mathcal{N}(K):=\left\{p: p \circ F_{K}=D F_{K}^{-T} \widehat{p}, \widehat{p} \in \mathcal{N}(\widehat{K})\right\}
$$

Let us remark that the mapping between $\mathcal{N}(K)$ and $\mathcal{N}(\widehat{K})$ is a kind of Piola transform for the "rot" operator, rot $p:=\partial p / \partial y-\partial p / \partial x$ and, in this case, there holds

$$
\int_{\ell} p \cdot \tau=\int_{\hat{\ell}} \hat{p} \cdot \widehat{\tau}
$$

for all edge $\ell$ of the element $K=F(\widehat{K})$, where $\tau$ is the unit vector tangential to $\ell$. Then, we define the space (which will be used to approximate the shear stress $\gamma$ )

$$
Z_{h}:=\left\{\psi \in H_{0}(\operatorname{rot}, \Gamma):\left.\psi\right|_{K} \in \mathcal{N}(K) \forall K \in \mathcal{T}_{h}^{\Gamma}\right\}
$$

that corresponds to the lowest-order rotated Raviart-Thomas space [19,21]. We remark that, for $Z_{h} \subset$ $H_{0}(\operatorname{rot}, \Gamma)$, the tangential component of a function in $Z_{h}$ must be continuous along interelement boundaries and vanish on $\partial \Gamma$. In fact, the integrals (15) of these tangential components are the degrees of freedom defining an element of $Z_{h}$.

We consider the reduction operator

$$
\Pi: H^{1}(\Gamma)^{2} \cap H_{0}(\operatorname{rot}, \Gamma) \longrightarrow Z_{h}
$$

locally defined for each $\psi \in H^{1}(\Gamma)^{2}$ by (see $[8,19]$ )

$$
\int_{\ell} \Pi \psi \cdot \tau=\int_{\ell} \psi \cdot \tau
$$

for every edge $\ell$ of the triangulation ( $\tau$ being a unit tangent vector along $\ell$ ). It can be shown that this operator satisfies $[8,19]$

$$
\|\psi-\Pi \psi\|_{0, \Gamma} \leq C h\|\psi\|_{1, \Gamma} .
$$

For the transverse displacements we take standard bilinear isoparametric elements, namely,

$$
W_{h}:=\left\{v_{h} \in H_{0}^{1}(\Gamma):\left.v_{h}\right|_{K} \in Q(K) \forall K \in \mathcal{T}_{h}^{\Gamma}\right\}
$$

where $Q(K):=\left\{p \in L^{2}(K): p \circ F_{K} \in Q_{1}(\widehat{K})\right\}$, for all $K \in \mathcal{T}_{h}$.

Finally, the finite element spaces for the rotations is defined by

$$
H_{h}:=\left\{\eta \in H_{0}^{1}(\Gamma)^{2}:\left.\eta\right|_{K} \in Q(K)^{2} \forall K \in \mathcal{T}_{h}^{\Gamma}\right\}
$$

The approximation of the plate problem by using the spaces $W_{h}, H_{h}, Z_{h}$, and the reduction operator $\Pi$, corresponds to the method MITC4.

We impose weakly the interface condition (4), because doing it strongly $\left(i . e ., u_{h} \cdot n=w_{h}\right.$ on $\Gamma$ ) is too stringent (see [5]). Let $\mathcal{C}_{h}:=\{\mathcal{F}: \mathcal{F}$ is a face of the fluid meshes lying on $\Gamma\}$. Then, we take as discrete space for the coupled problem

$$
\mathcal{V}_{h}:=\left\{\left(v_{h}, \eta_{h}, \phi_{h}\right) \in W_{h} \times H_{h} \times R_{h}: \phi_{h} \cdot n=0 \text { on } \Gamma_{\mathrm{R}} \text { and } \int_{\mathcal{F}} \phi_{h} \cdot n=\int_{\mathcal{F}} v_{h} \forall \mathcal{F} \in \mathcal{C}_{h}\right\} .
$$


The corresponding discrete eigenvalue problem is:

Find $\lambda_{h} \in \mathbb{R}$ and $0 \neq\left(w_{h}, \beta_{h}, u_{h}\right) \in \mathcal{V}_{h}$ such that

$$
\left\{\begin{array}{l}
a\left(\beta_{h}, \eta_{h}\right)+\frac{\kappa}{t^{2}} \int_{\Gamma}\left(\nabla w_{h}-\Pi \beta_{h}\right) \cdot\left(\nabla v_{h}-\Pi \eta_{h}\right)+\int_{\Omega} \hat{\rho}_{\mathrm{F}} c^{2} \operatorname{div} u_{h} \operatorname{div} \phi_{h} \\
=\lambda_{h}\left(\int_{\Gamma} \hat{\rho}_{\mathrm{P}} w_{h} v_{h}+\frac{t^{2}}{12} \int_{\Gamma} \hat{\rho}_{\mathrm{P}} \beta_{h} \cdot \eta_{h}+\int_{\Omega} \hat{\rho}_{\mathrm{F}} u_{h} \cdot \phi_{h}\right) \quad \forall\left(v_{h}, \eta_{h}, \phi_{h}\right) \in \mathcal{V}_{h} .
\end{array}\right.
$$

Note that the fact that $\mathcal{V}_{h} \not \subset \mathcal{V}$ and the use of the reduction operator $\Pi$ lead to two variational crimes for our method.

Analogously to the continuous case, $\lambda_{h}=0$ is an eigenvalue of this problem, with corresponding eigenspace

$$
\mathcal{K}_{h}:=\left\{\left(0,0, \phi_{h}\right) \in \mathcal{V}_{h}: \operatorname{div} \phi_{h}=0 \text { in } \Omega \text { and } \phi_{h} \cdot n=0 \text { on } \partial \Omega\right\}
$$

Hence, for the theoretical analysis, we may restrict the discrete eigenvalue problem to the space $\mathcal{G}_{h}$ given by the orthogonal complement of $\mathcal{K}_{h}$ in $\mathcal{V}_{h}$ with respect to $r_{t}$. We write

Find $\lambda_{h} \in \mathbb{R}$ and $0 \neq\left(w_{h}, \beta_{h}, u_{h}\right) \in \mathcal{G}_{h}$ such that

$$
s_{t h}\left(\left(w_{h}, \beta_{h}, u_{h}\right),\left(v_{h}, \eta_{h}, \phi_{h}\right)\right)=\lambda_{h} r_{t}\left(\left(w_{h}, \beta_{h}, u_{h}\right),\left(v_{h}, \eta_{h}, \phi_{h}\right)\right) \quad \forall\left(v_{h}, \eta_{h}, \phi_{h}\right) \in \mathcal{G}_{h}
$$

with

$$
s_{t h}\left(\left(w_{h}, \beta_{h}, u_{h}\right),\left(v_{h}, \eta_{h}, \phi_{h}\right)\right):=a\left(\beta_{h}, \eta_{h}\right)+\frac{\kappa}{t^{2}} \int_{\Gamma}\left(\nabla w_{h}-\Pi \beta_{h}\right) \cdot\left(\nabla v_{h}-\Pi \eta_{h}\right)+\int_{\Omega} \hat{\rho}_{\mathrm{F}} c^{2} \operatorname{div} u_{h} \operatorname{div} \phi_{h} .
$$

Because $\phi_{h}$ is not necessarily a gradient, for $\left(v_{h}, \eta_{h}, \phi_{h}\right) \in \mathcal{G}_{h}$, we have that $\mathcal{G}_{h} \not \subset \mathcal{G}$, and then a third variational crime for our methods.

To define the arises discrete analogue of the operator $T$, we need the following lemma which provides a Helmholtz decomposition for the discrete fluid displacements.

Lemma 3.1. For any $\left(v_{h}, \eta_{h}, \phi_{h}\right) \in \mathcal{G}_{h}, \phi_{h}$ can be written as

$$
\phi_{h}=\nabla \xi+\chi
$$

with $\xi$ and $\chi$ satisfying $\left(v_{h}, \eta_{h}, \nabla \xi\right) \in \mathcal{G}$ and $\operatorname{div} \chi=0$. Moreover, there exists a constant $C$, independent of $h$, such that

$$
\begin{aligned}
\|\nabla \xi\|_{1, \Omega} & \leq C\left(\left\|\operatorname{div} \phi_{h}\right\|_{0, \Omega}+\left\|v_{h}\right\|_{1, \Gamma}\right) \\
\|\chi\|_{0, \Omega} & \leq C h\left(\left\|\operatorname{div} \phi_{h}\right\|_{0, \Omega}+\left\|v_{h}\right\|_{1, \Gamma}\right)
\end{aligned}
$$

Proof. We do not include it here since it is essentially identical to those of Theorem 1.6.1 in [14].

As a consequence of the previous lemma, $\|\cdot\|$. and $\|\cdot\|$ are equivalent on $\mathcal{G}_{h}$ (with equivalence constants not depending on $h)$. On the other hand, $a\left(\beta_{h}, \eta_{h}\right)+\frac{\kappa}{t^{2}} \int_{\Gamma}\left(\nabla w_{h}-\Pi \beta_{h}\right) \cdot\left(\nabla v_{h}-\Pi \eta_{h}\right)$ is known to be uniformly coercive on $H_{h} \times W_{h}$ (see [10]). Then, clearly, the bilinear form $s_{t h}$ are coercive on $\mathcal{G}_{h}$, with coerciveness constant independent of $t$ and $h$. 
We introduce the following operator $T_{t h}$ by

$$
\begin{aligned}
T_{t h}: \mathcal{H} & \longrightarrow \mathcal{G}_{h} \\
(f, \theta, g) & \longmapsto\left(w_{h}, \beta_{h}, u_{h}\right)
\end{aligned}
$$

with $\left(w_{h}, \beta_{h}, u_{h}\right) \in \mathcal{G}_{h}$ being the solution of

$$
s_{t h}\left(\left(w_{h}, \beta_{h}, u_{h}\right),\left(v_{h}, \eta_{h}, \phi_{h}\right)\right)=r_{t}\left((f, \theta, g),\left(v_{h}, \eta_{h}, \phi_{h}\right)\right) \quad \forall\left(v_{h}, \eta_{h}, \phi_{h}\right) \in \mathcal{G}_{h} .
$$

These operators are uniformly bounded in $t$ and $h$. Moreover, the non-zero eigenvalues $\mu_{h}$ of $T_{t h}$ are related with the eigenvalues $\lambda_{h}$ of Problem (19) by $\mu_{h}=\frac{1}{\lambda_{h}}$.

\section{Convergence of the Discrete operators}

We are going to prove that the operator $T_{t h}$ converge to $T_{t}$ in norm as $h$ goes to zero, in both $\|\cdot\|$ and the norm induced by $r_{t}(\cdot, \cdot)$. This fact will be used in the next section to prove the spectral convergence.

From now on and throughout this section, we consider $(f, \theta, g) \in \mathcal{H}$ fixed and denote

$$
\begin{array}{ll}
(w, \beta, u):=T_{t}(f, \theta, g), & \left(w_{h}, \beta_{h}, u_{h}\right):=T_{t h}(f, \theta, g), \\
\gamma:=\frac{\kappa}{t^{2}}(\nabla w-\beta), & \gamma_{h}:=\frac{\kappa}{t^{2}}\left(\nabla w_{h}-\Pi \beta_{h}\right) .
\end{array}
$$

From (22) and the definition of $s_{t}$ we have

$$
\begin{aligned}
a\left(\beta-\beta_{h}, \eta_{h}\right)+\int_{\Gamma}\left(\gamma-\gamma_{h}\right) \cdot\left(\nabla v_{h}-\Pi \eta_{h}\right)+ & \int_{\Omega} \hat{\rho}_{\mathrm{F}} c^{2} \operatorname{div}\left(u-u_{h}\right) \operatorname{div} \phi_{h} \\
& =\int_{\Gamma} \gamma \cdot\left(\eta_{h}-\Pi \eta_{h}\right)+M_{h}\left(v_{h}, \eta_{h}, \phi_{h}\right) \quad \forall\left(v_{h}, \eta_{h}, \phi_{h}\right) \in \mathcal{G}_{h}
\end{aligned}
$$

where

$$
M_{h}\left(v_{h}, \eta_{h}, \phi_{h}\right):=s_{t}\left((w, \beta, u),\left(v_{h}, \eta_{h}, \phi_{h}\right)\right)-r_{t}\left((f, \theta, g),\left(v_{h}, \eta_{h}, \phi_{h}\right)\right) .
$$

Note that, two consistency terms appear in the error equation. The first one due of the use of the reduction operator $\Pi$ in $s_{t h}$ and the last one because the space $\left.\mathcal{G}_{h} \not \subset \mathcal{G}\right)$.

The argument to prove that the consistency terms are bounded and the corresponding convergence of the operators, have been used in [12] for similar methods on triangular and tetrahedral meshes. The proof are essentially identical to those of that reference. However, for the sake of completeness, we include some of these.

By using (17), we can easily estimate the term $\int_{\Gamma} \gamma \cdot\left(\eta_{h}-\Pi \eta_{h}\right)$ in the equation above. For the second consistency term we have:

Lemma 4.1. There holds

$$
\left|M_{h}\left(v_{h}, \eta_{h}, \phi_{h}\right)\right| \leq C h\|g\|_{0, \Omega}\left\|\left(v_{h}, \eta_{h}, \phi_{h}\right)\right\|_{\bullet} \quad \forall\left(v_{h}, \eta_{h}, \phi_{h}\right) \in \mathcal{G}_{h} .
$$

Proof. See Lemma 5.1 in [12].

Now, we prove that the spaces $\mathcal{G}_{h}$ provide suitable approximations for $(w, \beta, u)$ :

Lemma 4.2. There exists $(\hat{w}, \hat{\beta}, \hat{u}) \in \mathcal{G}_{h}$ such that

$$
\|(\hat{w}, \hat{\beta}, \hat{u})-(w, \beta, u)\| \leq C h|(f, \theta, g)|_{t} .
$$


Moreover, if $\hat{\gamma}:=\frac{\kappa}{t^{2}}(\nabla \hat{w}-\Pi \hat{\beta})$, the following estimate also holds

$$
t\|\hat{\gamma}-\gamma\|_{0, \Gamma} \leq C h|(f, \theta, g)|_{t} .
$$

Proof. According with Theorems 3.7 and 3.1 in [13], there exist $\hat{\beta} \in H_{h}$ and an operator $\widetilde{\Pi}: H_{0}(\operatorname{rot}, \Gamma) \cup$ $H^{1}(\Gamma)^{2} \longrightarrow Z_{h}$ such that

$$
\|\hat{\beta}-\beta\|_{1, \Gamma} \leq C h\|\beta\|_{2 . \Gamma}
$$

and

$$
\operatorname{rot}\left(\Pi \hat{\beta}-\frac{t^{2}}{\kappa} \widetilde{\Pi} \gamma\right)=0
$$

By virtue of the last equality and Lemma 2.1 in that paper, there exist $\hat{w} \in W_{h}$ such that $\nabla \hat{w}=\frac{t^{2}}{\kappa} \widetilde{\Pi} \gamma-\Pi \hat{\beta}$. Then for $\hat{\gamma}=\widetilde{\Pi} \gamma=\frac{\kappa}{t^{2}}(\nabla \hat{w}-\Pi \hat{\beta})$ we have $\|\hat{\gamma}-\gamma\|_{0, \Gamma} \leq C h\|\gamma\|_{1, \Gamma}$.

On the other hand, because

$$
\nabla(w-\hat{w})=\frac{t^{2}}{\kappa}(\gamma-\hat{\gamma})+\Pi \hat{\beta}-\beta=\frac{t^{2}}{\kappa}(\gamma-\hat{\gamma})+\Pi(\hat{\beta}-\beta)+(\Pi \beta-\beta)
$$

by using (17) and the previous estimates we have $\|\hat{w}-w\|_{1, \Gamma} \leq C h\left(\|\gamma\|_{1, \Gamma}+\|\beta\|_{2, \Gamma}\right)$.

Arguing as in Theorem 5.2 of [7] we can find $u^{I} \in R_{h}$ such that $\left(\hat{w}, \hat{\beta}, u^{I}\right) \in V_{h}$ and $\left\|u^{I}-u\right\|_{H(\operatorname{div}, \Omega)} \leq$ $C h\left[\|w\|_{2, \Gamma}+\|u\|_{H^{1}(\mathrm{div}, \Omega)}\right]$.

Now, let $\left(0,0, u_{\mathcal{K}_{h}}\right)$ be the $r_{t}$ projection of $\left(\hat{w}, \hat{\beta}, u^{I}\right)$ onto $\mathcal{K}_{h}$. Hence, for $\hat{u}:=u^{I}-u_{\mathcal{K}_{h}},(\hat{w}, \hat{\beta}, \hat{u}) \in \mathcal{G}_{h}$. Moreover, since $u_{\mathcal{K}_{h}}$ and $(\hat{u}-u)$ are orthogonal in $H(\operatorname{div}, \Omega)$, we have

$$
\|\hat{u}-u\|_{H(\operatorname{div}, \Omega)} \leq\left\|(\hat{u}-u)+u_{\mathcal{K}_{h}}\right\|_{H(\operatorname{div}, \Omega)}=\left\|u^{I}-u\right\|_{H(\operatorname{div}, \Omega)} .
$$

Therefore, by applying the a priori estimate in Theorem 2.1 we conclude the proof.

The following lemma establishes convergence for the discrete operators in $\|\cdot\|_{.}$. As a byproduct we obtain convergence for the shear strains, which will be used in the next section.

Lemma 4.3. There holds

$$
\left\|w-w_{h}\right\|_{1, \Gamma}+\left\|\beta-\beta_{h}\right\|_{1, \Gamma}+t\left\|\gamma-\gamma_{h}\right\|_{0, \Gamma}+\left\|\operatorname{div}\left(u-u_{h}\right)\right\|_{0, \Omega} \leq C h|(f, \theta, g)|_{t} .
$$

Proof. See Lemma 5.3 in [12].

Now, we may prove the claimed convergence:

Theorem 4.4. There exists a constant $C$ such that, for any $(f, \theta, g) \in \mathcal{H}$, there holds

$$
\left\|\left(T_{t}-T_{t h}\right)(f, \theta, g)\right\| \leq C h|(f, \theta, g)|_{t} .
$$

Proof. The theorem is an immediate consequence of Lemma 4.3 and Lemma 5.4 in [12]. 
Finally, we obtain the following estimate:

Theorem 4.5. There exists a constant $C$ such that, for any $(f, \theta, g) \in \mathcal{H}$, there holds

$$
\left|r_{t}\left(\left(T_{t}-T_{t h}\right)(f, \theta, g),(f, \theta, g)\right)\right| \leq C h^{2}|(f, \theta, g)|_{t}
$$

Proof. We consider a decomposition of $u_{h}$ according to lemma $3.1 ;$ i.e., $u_{h}=\nabla \xi+\chi$. Recalling the equation (6.2) in [12] we have

$$
\begin{aligned}
r_{t}\left(\left(T_{t}-T_{t h}\right)(f, \theta, g),(f, \theta, g)\right)= & a\left(\beta-\beta_{h}, \beta-\beta_{h}\right)+\frac{t^{2}}{\kappa} \int_{\Gamma}\left|\gamma-\gamma_{h}\right|^{2}+\int_{\Omega} \hat{\rho}_{\mathrm{F}} c^{2}\left(\operatorname{div} u-\operatorname{div} u_{h}\right)^{2} \\
& -2 \int_{\Omega} \hat{\rho}_{\mathrm{F}} g \cdot \chi-2 \int_{\Gamma} \gamma \cdot\left(\beta_{h}-\Pi \beta_{h}\right) .
\end{aligned}
$$

Because of continuity of $a(\cdot, \cdot)$ and Lemma 4.3, there only remains to estimate the two last terms in the right hand side of the equation above.

The proof in Lemmas 4.2 and 4.3 in [13] can be easily adapted to prove the estimate for the last term. In fact, this term has been analyzed in that paper in order to obtain optimal $L^{2}$ error estimate for the MITC4 methods for a clamped plate.

On the other hand, since $(f, \theta, g) \in \mathcal{G}$, then $g=\nabla q$ and, because of $(9), q \in H^{2}(\Omega)^{2}$ with

$$
\|q\|_{2, \Omega} \leq C\left(\|f\|_{1 / 2, \Gamma}+\|\operatorname{div} g\|_{0, \Omega}\right) \leq C\|(f, \theta, g)\| .
$$

Now, since $\operatorname{div} \chi=0$ and $\chi=u_{h}-\nabla \xi$, we have

$$
\int_{\Omega} \hat{\rho}_{\mathrm{F}} g \cdot \chi=\int_{\partial \Omega} \hat{\rho}_{\mathrm{F}} q\left(u_{h}-\nabla \xi\right) \cdot n=\int_{\Gamma} \hat{\rho}_{\mathrm{F}} q\left(u_{h} \cdot n-w_{h}\right),
$$

the latter because of $\left(w_{h}, \beta_{h}, \nabla \xi\right) \in \mathcal{G}$. Since $\left(w_{h}, \beta_{h}, u_{h}\right) \in \mathcal{G}_{h}$, then $P\left(u_{h} \cdot n\right)=P\left(w_{h}\right)$, with $P$ being the $L^{2}(\Gamma)$-projection onto the piecewise constant functions on $\mathcal{C}_{h}$. Hence,

$$
\begin{aligned}
\left|\int_{\Omega} \hat{\rho}_{\mathrm{F}} g \cdot \chi\right| & =\left|\int_{\Gamma} \hat{\rho}_{\mathrm{F}}[q-P(q)]\left[u_{h} \cdot n-P\left(u_{h} \cdot n\right)+P\left(w_{h}\right)-w_{h}\right]\right| \\
& \leq\|q-P(q)\|_{0, \Gamma}\left(\left\|u_{h} \cdot n-P\left(u_{h} \cdot n\right)\right\|_{0, \Gamma}+\left\|P\left(w_{h}\right)-w_{h}\right\|_{0, \Gamma}\right) \\
& \leq C h\|q\|_{1, \Gamma}\left(\left\|u_{h} \cdot n-P\left(u_{h} \cdot n\right)\right\|_{0, \Gamma}+\left\|P\left(w_{h}\right)-w_{h}\right\|_{0, \Gamma}\right),
\end{aligned}
$$

and $\|q\|_{1, \Gamma} \leq C\|q\|_{2, \Omega} \leq C\|(f, \theta, g)\|$. Then, we estimates the remainder two terms. The proof of Lemma 1.6.8 in [14] can be easily adapted to prove that

$$
\left\|u_{h} \cdot n-P\left(u_{h} \cdot n\right)\right\|_{0, \Gamma} \leq C h\|(f, \theta, g)\| .
$$

For the last term, we have

$$
\begin{aligned}
\left\|P\left(w_{h}\right)-w_{h}\right\|_{0, \Gamma} & \leq\left\|P\left(w_{h}-w\right)\right\|_{0, \Gamma}+\|P w-w\|_{0, \Gamma}+\left\|w-w_{h}\right\|_{0, \Gamma} \\
& \leq\|P w-w\|_{0, \Gamma}+C\left\|w-w_{h}\right\|_{0, \Gamma} \\
& \leq C h\|w\|_{1, \Gamma}+C h\|(f, \theta, g)\| \\
& \leq C h\|(f, \theta, g)\|,
\end{aligned}
$$

where we have used Lemmas 2.1 and 4.3. Thus, we conclude proof. 
Remark 4.6. According to [13], the macroelement assumption on the mesh $\mathcal{T}_{h}^{\Gamma}$ for the MITC4 method is only used to prove an optimal order estimate for the last terms in the right hand side of the equation (26) in the proof of the theorem above. However, a modification of this method is introduced in this reference.It consists of enriching the discrete space $H_{h}$ by means of a rotation of a space used for the approximation of the Stokes problem. For this methods, which is called DL4, the macroelement assumption is not necessary.

\section{Spectral approximation}

It is shown in [12], that as the thickness $t \rightarrow 0$, each eigenvalue $\mu$ of problem (6) converge to some limit $\mu_{0}$. Indeed, $\mu_{0}$ are the eigenvalues of the operator associated with the classical Kirchhoff model of the same plate coupled with the fluid (see Th. 3.2 in [12]). From now on, for simplicity, we assume that $\mu$ is an eigenvalue of $T_{t}$ which converges to a simple eigenvalue $\mu_{0}$ as $t$ goes to zero (see Sect. 3 in [12] for further discussions).

As a consequence of Theorem 4.4, for each simple eigenvalue $\mu$ of $T_{t}$, there is exactly one eigenvalue $\mu_{h}$ of $T_{t h}$ converging to $\mu$ as $h$ goes to zero (see for instance [12]). The following theorem shows optimal $t$-independent error estimates:

Theorem 5.1. Let $\mu$ and $\mu_{h}$ be simple eigenvalues of $T_{t}$ and $T_{t h}$, respectively, such that $\mu_{h} \rightarrow \mu$ as $h \rightarrow 0$. Let $(w, \beta, u)$ and $\left(w_{h}, \beta_{h}, u_{h}\right)$ be the eigenfunctions corresponding to $\mu$ and $\mu_{h}$, respectively, both normalized in the same manner. Then, there exists $C>0$ such that, for $t$ and $h$ small enough, there holds

$$
\left\|(w, \beta, u)-\left(w_{h}, \beta_{h}, u_{h}\right)\right\| \leq C h,
$$

and

$$
\left|\mu_{t}-\mu_{t h}\right| \leq C h^{2} .
$$

Proof. The proof, which relies on Theorems 4.4 and 4.5, are essentially the same as those of Theorems 6.2 and 6.3 in [12].

\section{NumERICAL EXPERIMENTS}

In this section we present numerical results obtained with a implementation of the method.

We have tested the methods by reproducing the experiments in [12]. In that paper, the problem is approximated by using triangular and tetrahedral finite elements; more precisely, using DL3 for plate and hexahedral Raviart-Thomas elements for the fluid.

We remark that it is well know that, from the point of view of efficiency, for the same number of degrees of freedom, hexahedral element approach the exact solution better than tetrahedral ones for structural problems (see for instance [6]). In fact, using Raviart-Thomas elements, the number of faces (degree of freedom for this element) for hexahedral approximation is about one quarter that for tetrahedrical ones, on meshes with same vertices (see [6]).

We have considered a steel 3D cavity completely filled with water with all of its walls being perfectly rigid, except for one of them which is an elastic plate. The geometric data are given in Figure 3. The physical parameters of plate and fluid are the following ones:

- density of the plate: $\rho_{\mathrm{P}}=7700 \mathrm{~kg} / \mathrm{m}^{3}$;

- Young modulus: $E=1.44 \times 10^{11} \mathrm{~Pa}$;

- Poisson coefficient: $\nu=0.35$;

- density of the fluid: $\rho_{\mathrm{F}}=1000 \mathrm{~kg} / \mathrm{m}^{3}$;

- sound speed: $c=1430 \mathrm{~m} / \mathrm{s}$.

The method have been used on several successively refinements of the initial mesh (see Fig. 4). The refinement parameter $N$ stand for the number of layer of element for the fluid domain in the vertical direction. The number of layers in the other two direction being $2 N$ and $3 N$, as show the Figure 4. 


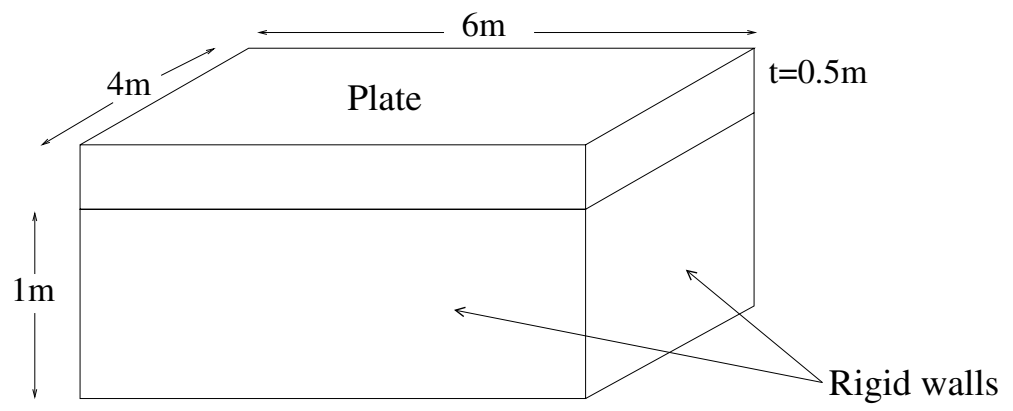

FIGURE 3. Cavity filled with fluid.

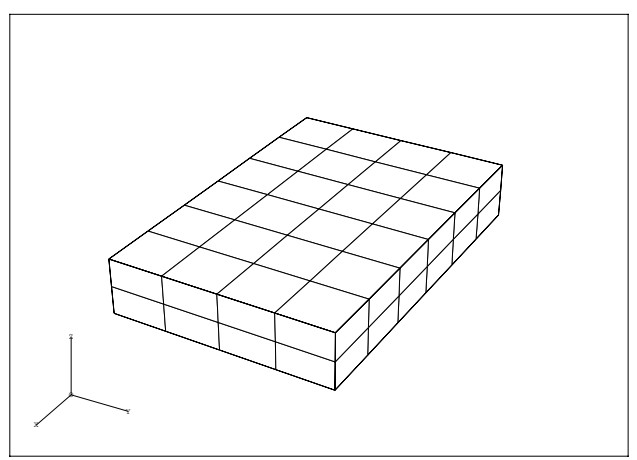

Figure 4. Fluid mesh with $N=2$.

TABLE 1. Angular vibration frequencies of a moderately thick steel plate in contact with water.

\begin{tabular}{|c|c|c|c||c||c|}
\hline Mode & $N=4$ & $N=5$ & $N=6$ & $\alpha$ & $\omega_{m}$ \\
\hline$\omega_{1}^{h}$ & 703.4418 & 701.3463 & 700.1963 & 1.95 & 697.5025809 \\
$\omega_{2}^{h}$ & 1046.7326 & 1035.0140 & 1028.7364 & 2.07 & 1015.0498050 \\
$\omega_{3}^{h}$ & 1096.3214 & 1091.2521 & 1088.4428 & 1.90 & 1081.6537906 \\
$\omega_{4}^{h}$ & 1329.5584 & 1325.0901 & 1322.6234 & 1.92 & 1316.7369551 \\
$\omega_{5}^{h}$ & 1513.5155 & 1492.4589 & 1481.2156 & 2.08 & 1456.8201771 \\
\hline
\end{tabular}

We have computed approximations of the free vibration angular frequencies corresponding to the lowestfrequency vibration modes of the coupled system.

Table 1 shows the five lowest vibration frequencies computed by our method for the plate coupled with water. The table includes also the value of the vibration frequencies obtained by extrapolating the computed ones $\left(\omega_{m}^{h}\right)$ as well as the estimated order of convergence $\alpha$. Such values have been obtained by means of a least square fitting of the model

$$
\omega_{m}^{h} \approx \omega_{m}\left(1+C_{m} h^{\alpha}\right)
$$

for the frequencies calculated on three different meshes $(N=4,5,6)$.

The obtained result compare perfectly well with those in $[6,12]$.

Figures 5 to 9 show the deformed plate and the fluid pressure for each of these five vibration modes.

According to [12], we also check the stability of the method as the thickness becomes small. Tables 2 and 3 show the results obtained for the first and second frequency vibration modes, for plates with different thicknesses. 
TABLE 2. First vibration frequency $\omega_{1}^{h}$ for plates of different thickness coupled with fluid.

\begin{tabular}{|c|c|c|c||c||c|}
\hline$t$ & $N=4$ & $N=5$ & $N=6$ & $\alpha$ & $\omega_{1}$ \\
\hline 0.5 & 703.4418 & 701.3463 & 700.1963 & 1.95 & 697.5025809 \\
0.05 & 747.5121 & 746.6361 & 746.1595 & 1.99 & 745.0693080 \\
0.005 & 747.5326 & 746.6569 & 746.1806 & 1.99 & 745.0908998 \\
0.0005 & 747.5328 & 746.6571 & 746.1808 & 1.99 & 745.0911159 \\
\hline
\end{tabular}

TABLE 3. Second vibration frequency $\omega_{2}^{h}$ for plates of different thickness coupled with fluid.

\begin{tabular}{|c|c|c|c||c||c|}
\hline$t$ & $N=4$ & $N=5$ & $N=6$ & $\alpha$ & $\omega_{1}$ \\
\hline 0.5 & 1046.7326 & 1035.0140 & 1028.7364 & 2.07 & 1015.0498050 \\
0.05 & 1128.5113 & 1125.7410 & 1124.2345 & 1.99 & 1120.7874465 \\
0.005 & 1128.5296 & 1125.7600 & 1124.2539 & 1.99 & 1120.8077413 \\
0.0005 & 1128.5298 & 1125.7602 & 1124.2541 & 1.99 & 1120.8079442 \\
\hline
\end{tabular}
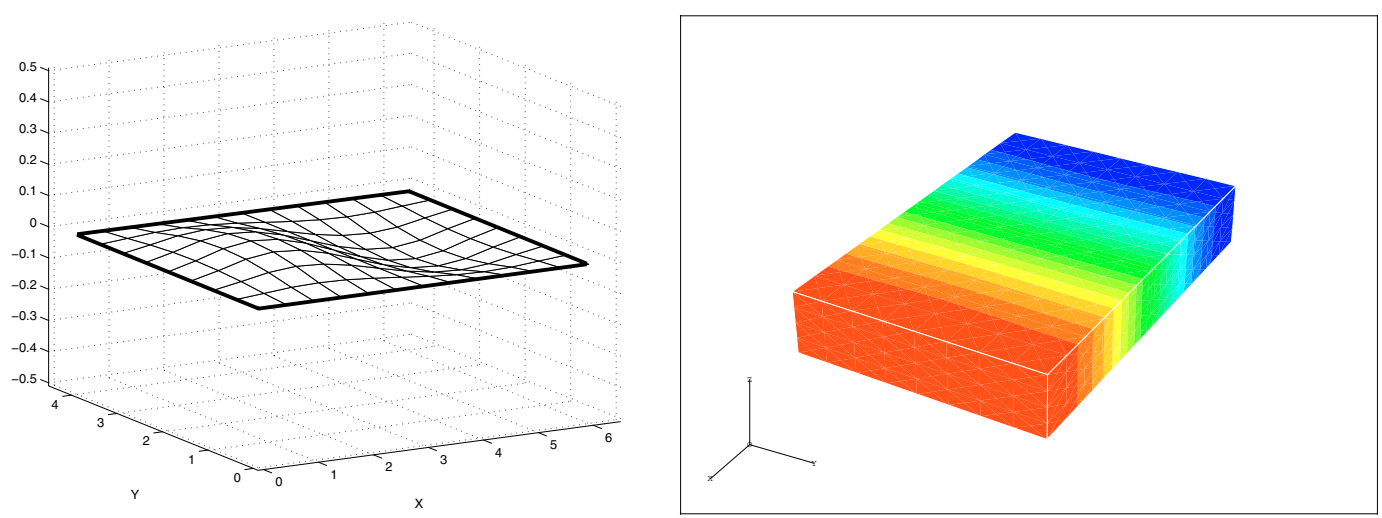

FIGURE 5. Deformed plate and fluid pressure for the mode $\omega_{1}$.

To allow for comparison we scale the frequencies by using the assumption made in Section 2 on the densities of the plate and fluid (namely, $\rho_{\mathrm{F}}=\hat{\rho}_{\mathrm{F}} t^{3}$ and $\rho_{\mathrm{P}}=\hat{\rho}_{\mathrm{P}} t^{2}$ ). Note that the convergence behavior does not depend on the thickness. 

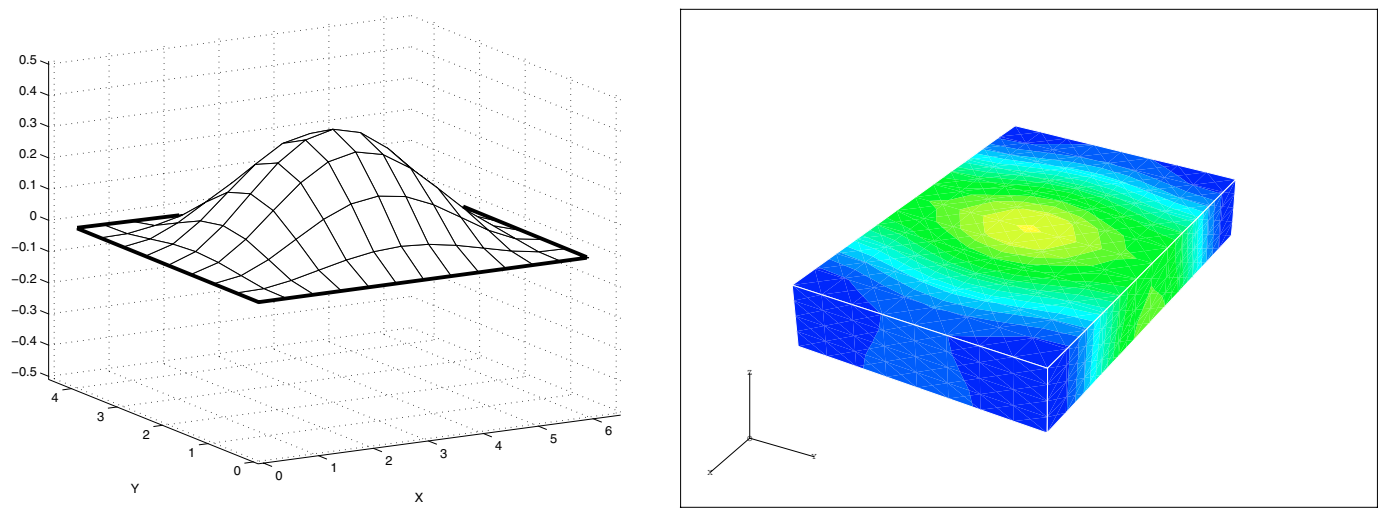

Figure 6. Deformed plate and fluid pressure for the mode $\omega_{2}$.
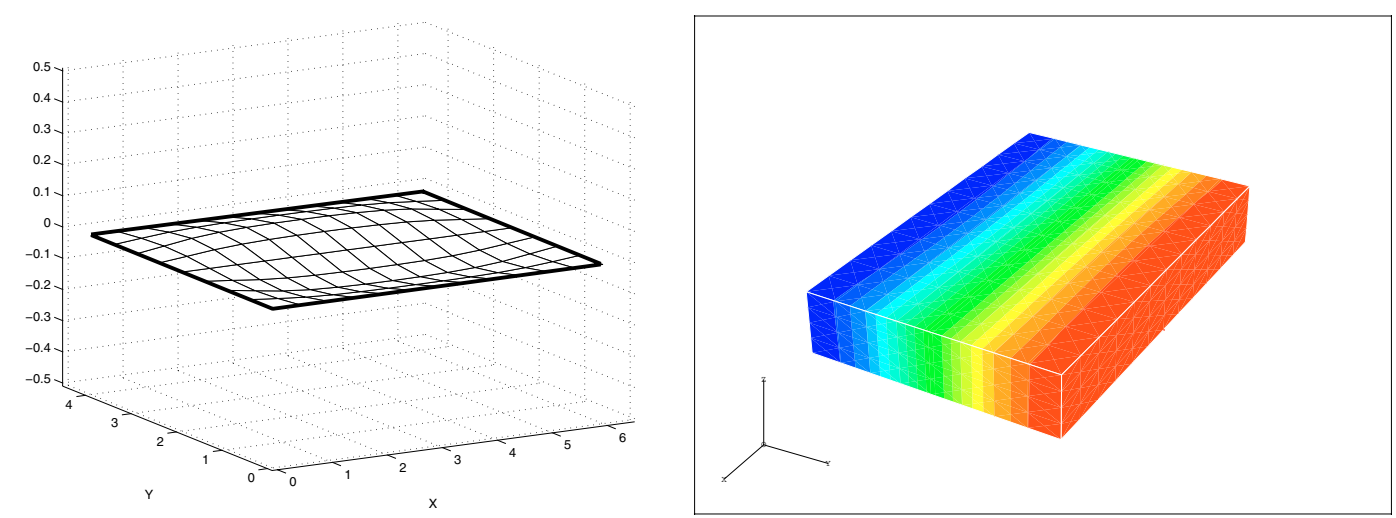

FiguRE 7. Deformed plate and fluid pressure for the mode $\omega_{3}$.
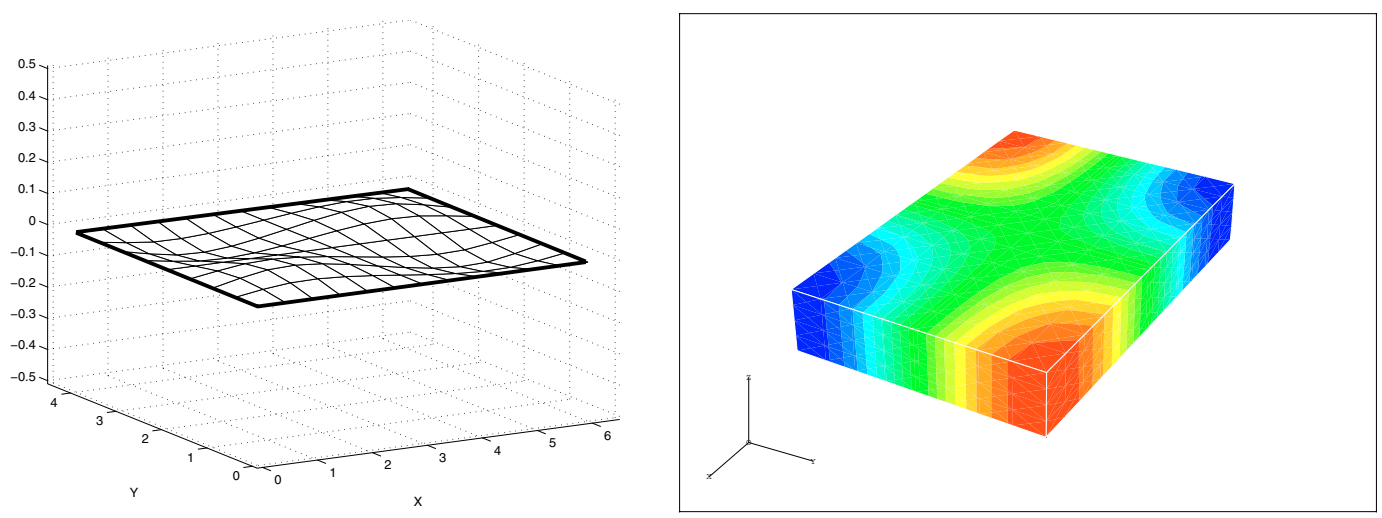

Figure 8. Deformed plate and fluid pressure for the mode $\omega_{4}$.

Acknowledgements. The author wants to thank Dr. Rodolfo Rodríguez from Universidad de Concepción, Chile, for his helpful discussions and comments. 

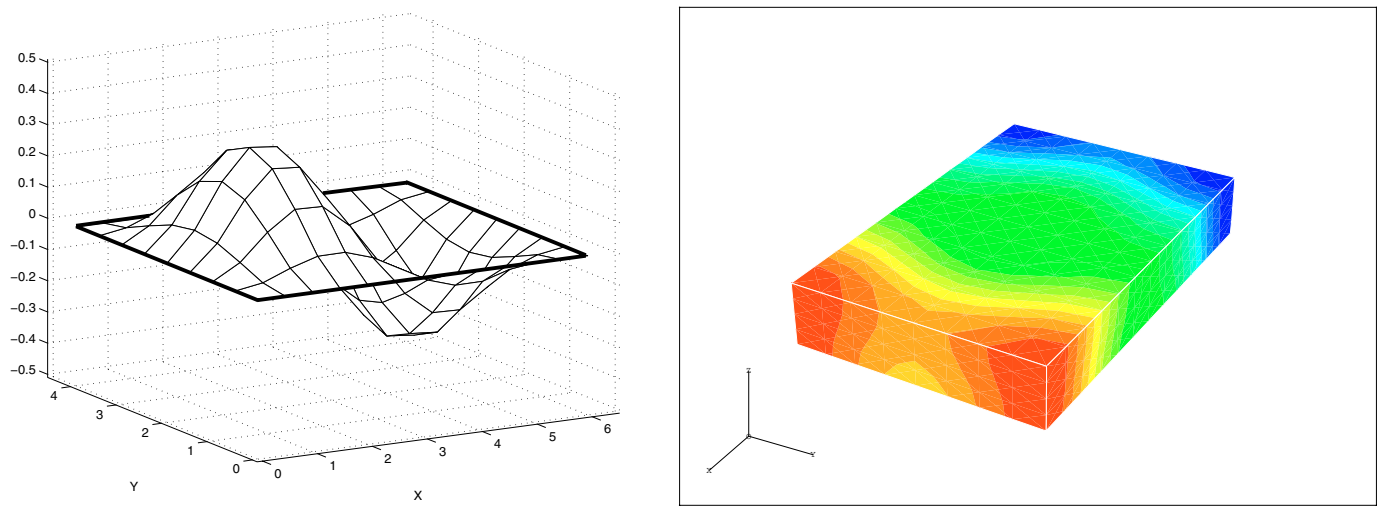

Figure 9. Deformed plate and fluid pressure for the mode $\omega_{5}$.

\section{REFERENCES}

[1] S.M. Alessandrini, D.N. Arnold, R.S. Falk and A.L. Madureira, Derivation and justification of plate models by variational methods, in Plates and Shells, M. Fortin Ed., AMS, Providence, CRM Proc. Lect. Notes Ser. 21 (1999) 1-20.

[2] D.N. Arnold and R.S. Falk, A uniformly accurate finite element method for the Reissner-Mindlin plate. SIAM J. Numer. Anal. 26 (1989) 1276-1290.

[3] K.J. Bathe and F. Brezzi, On the convergence of a four-node plate bending element based on Mindlin/Reissner plate theory and a mixed interpolation in Mathematics of Finite Elements an Applications V, J.R. Whiteman Ed., Academic Press, London (1985) 491-503.

[4] K.J. Bathe and E.N. Dvorkin, A four-node plate bending element based on Mindlin/Reissner plate theory and a mixed interpolation. Int. J. Numer. Methods Eng. 21 (1985) 367-383.

[5] A. Bermúdez and R. Rodríguez, Finite element computation of the vibration modes of a fluid-solid system. Comp. Methods Appl. Mech. Eng. 119 (1994) 355-370.

[6] A. Bermúdez, P. Gamallo and R. Rodríguez, An hexahedral face element for elastoacoustic vibration problems. J. Comp. Acoust. 119 (1994) 355-370.

[7] A. Bermúdez, R. Durán, M.A. Muschietti, R. Rodríguez and J. Solomin, Finite element vibration analysis of fluid-solid systems without spurious modes. SIAM J. Numer. Anal. 32 (1995) 1280-1295.

[8] F. Brezzi and M. Fortin, Mixed and Hybrid Finite Element Methods. Springer-Verlag, New York (1991).

[9] F. Brezzi, M. Fortin and R. Stenberg, Quasi-optimal error bounds for approximation of shear-stresses in Mindlin-Reissner plate models. Math. Models Methods Appl. Sci. 1 (1991) 125-151.

[10] R. Durán and E. Liberman, On mixed finite element methods for the Reissner-Mindlin plate model. Math. Comp. 58 (1992) 561-573.

[11] R. Durán, L. Hervella-Nieto, E. Liberman, R. Rodríguez and J. Solomin, Approximation of the vibration modes of a plate by Reissner-Mindlin equations. Math. Comp. 68 (1999) 1447-1463.

[12] R. Durán, L. Hervella-Nieto, E. Liberman, R. Rodríguez and J. Solomin, Finite element analysis of the vibration problem of a plate coupled with a fluid. Numer. Math. 86 (2000) 591-616.

[13] R. Durán, E. Hernández, L. Hervella-Nieto, E. Liberman and R. Rodríguez, Computation of the vibration modes of plates and shells by low-order MITC quadrilateral finite elements. SIAM J. Numer. Anal. 41 (2003) 1751-1772.

[14] P. Gamallo, Métodos numéricos de elementos finitos en problemas de interacción fluido-estructura. Ph.D. Thesis, U. de Santiago de Compostela, Spain (2002).

[15] V. Girault and P.A. Raviart, Finite Element Methods for Navier-Stokes Equations. Springer-Verlag, Berlin, Heidelberg, New York, Tokyo (1986).

[16] T.J.R. Hughes, The Finite Element Method: Linear Static and Dinamic Finite Element Analysis. Prentice-Hall, Englewood Cliffs, NJ (1987).

[17] H.J.-P. Morand and R. Ohayon, Fluid-structure interactions. John Wiley \& Sons, New York (1995).

[18] R. Rodríguez and J. Solomin, The order of convergence of eigenfrequencies in finite element approximations of fluid-structure interaction problems. Math. Comp. 65 (1996) 1463-1475.

[19] P.A. Raviart and J.M. Thomas, A mixed finite element method for second order elliptic problems, in Mathematical Aspects of Finite Element Methods, Springer-Verlag, Berlin, Heidelberg, New York. Lect. Notes Math. 606 (1977) 292-315.

[20] R. Stenberg and M. Suri, An $h p$ error analysis of MITC plate elements. SIAM J. Numer. Anal. 34 (1997) 544-568.

[21] J.M. Thomas, Sur l'analyse numérique des méthodes d'éléments finis hybrides et mixtes. Thèse de Doctorat d'Etat, Université Pierre et Marie Curie, Paris 6, France (1977). 